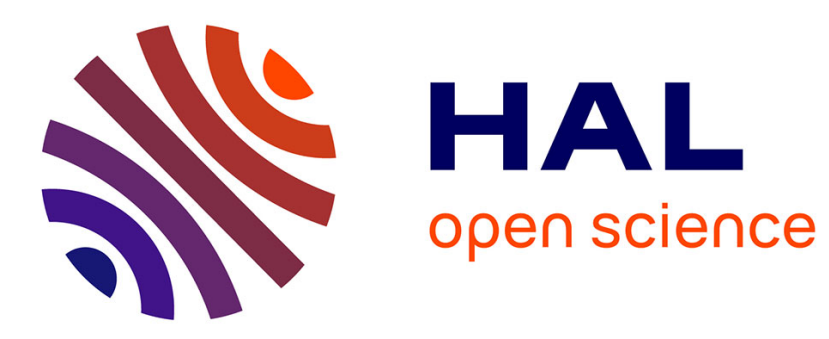

\title{
A New Rotation-Invariant Approach for Texture Analysis
}

\author{
Izem Hamouchene, Saliha Aouat
}

\section{To cite this version:}

Izem Hamouchene, Saliha Aouat. A New Rotation-Invariant Approach for Texture Analysis. 5th International Conference on Computer Science and Its Applications (CIIA), May 2015, Saida, Algeria. pp.45-53, 10.1007/978-3-319-19578-0_4 . hal-01789958

\section{HAL Id: hal-01789958 \\ https://hal.inria.fr/hal-01789958}

Submitted on 11 May 2018

HAL is a multi-disciplinary open access archive for the deposit and dissemination of scientific research documents, whether they are published or not. The documents may come from teaching and research institutions in France or abroad, or from public or private research centers.
L'archive ouverte pluridisciplinaire HAL, est destinée au dépôt et à la diffusion de documents scientifiques de niveau recherche, publiés ou non, émanant des établissements d'enseignement et de recherche français ou étrangers, des laboratoires publics ou privés. 


\title{
A new rotation-invariant approach for texture analysis
}

\author{
Izem Hamouchene and Saliha Aouat \\ Artificial Intelligence Laboratory (LRIA), Computer science Department \\ University of sciences and technology (USTHB), Algiers, Algeria \\ \{ihamouchene, saouat\}@usthb.dz
}

\begin{abstract}
Image processing and pattern recognition are one of the most important area of research in computer science. Recently, several studies have been made and efficient approaches have been proposed to provide efficient solutions to many real and industrial problems. Texture analysis is a fundamental field of image processing because all surfaces of objects are textured in nature. Thus, we proposed a new texture analysis method. In this paper, we proposed a novel texture analysis approach based on a recent feature extraction method called neighbor based binary pattern (NBP). The NBP method extract the local micro texture and is robust against rotation, which is a key problem in image processing. The proposed system extract two-reference NBP histograms from the texture in order to calculate a model of the texture. Finally, several models have been constructed to be able to recognize textures even after rotation. Textured images from Brodatz album database were used in the evaluation. Experimental studies have illustrated that the proposed system obtain very encouraging results robust to rotation compared to classical method.
\end{abstract}

Keywords: Rotation invariance, Texture analysis, Feature extraction, Neighbor based binary pattern.

\section{Introduction}

Texture analysis is one of fundamental domain in image processing and computer vision. In today's world, automatic image processing without human intervention has become an active research area. In fact, there is not a strict definition of the texture, but the texture can be defined as a visual pattern composed of entities that have characteristic such as brightness, color, shape, size, etc. Texture is present in most of real life objects in nature. This makes it fundamental and essential to analyze images. Texture can be subdivided into coarse, micro, macro, regular, periodic, aperiodic, random and stochastic type [1].

Texture analysis has been presented by Haralick [5]. Different approaches have been developed structural, statistical and transformed based approach. These approaches have been applied in different, various and recent applications such as face recognition [2], Fingerprint matching [3] and image segmentation [4]. Textured images are analyzed by identifying the local and global properties of the images. One of the key problem of image analysis is rotation. Indeed, how recognize a researched texture even after rotation. The rotation invariant problem remains unsolved today. In 
this study, we proposed a new system robust against rotation and extract pertinent patterns of the texture.

This paper is organized as follows: The next section we explain the recent feature extraction method applied on the proposed system, the neighbor based binary pattern (NBP). In section 3 we present the architecture of our proposed system. Section 4 illustrates experimental results using the proposed system and the last section conclude the paper.

\section{Neighbor based Binary Pattern}

The neighbor based binary pattern (NBP) is a very simple and efficient method to describe the texture. The NBP method was proposed for the first time by Izem et al. [6] [7]. This method was inspired by a famous feature extraction operator called Local Binary Pattern (LBP) [8] [9]. The important advantage of the LBP operator is its monotonic gray-scale transformation invariance [10] [11] [12] and its computational simplicity which makes it able to analyze an image in a very short time.

The idea of the NBP method is to consider one analysis window of $3 \times 3$ pixels. Each neighbor of the central pixel is thresholded by the next neighbor. Thus, if the central pixel is a noise it is not a problem because the value of the central pixel is not considered. In the other hand, if one neighbor is a noise, not all the pattern will be wrong but only 1 bit. . This minimizes the error rate of the recognition. So, each neighbor is encoded by the value 1 if its value is greater than the next neighbor is and 0 otherwise. The binary code is interpreted as a decimal number and represent the value of the central pixel in the NBP number. This process is illustrated in Fig. 1

Analysis window

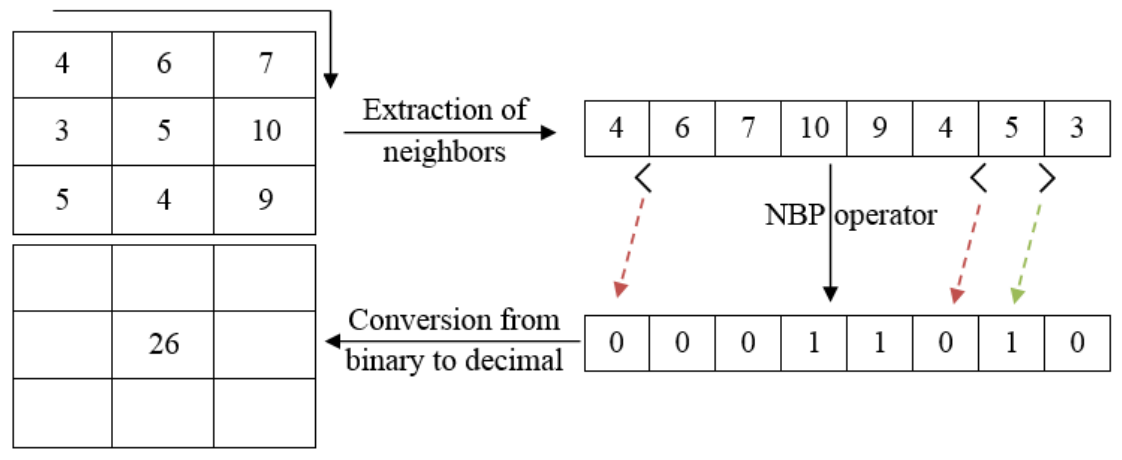

NBP Image

Fig. 1. Neighbor based Binary Pattern.

Fig. 1. Illustrates the obtained NBP value using the NBP method. The first neighbor (value 4) is less than the second (value 6). Thus, the first neighbor is encoded by the value 0 . After that, the obtained binary code is converted into a decimal number. 
Because it is difficult to find a general parametric model for this distribution, the features of the obtained NBP image are approximated by a two dimensional discrete patterns histogram. This histogram is created to collect up the occurrences of each pattern. The obtained histogram is used to describe the texture as show in Fig. 2. Usually, the histograms are normalized.

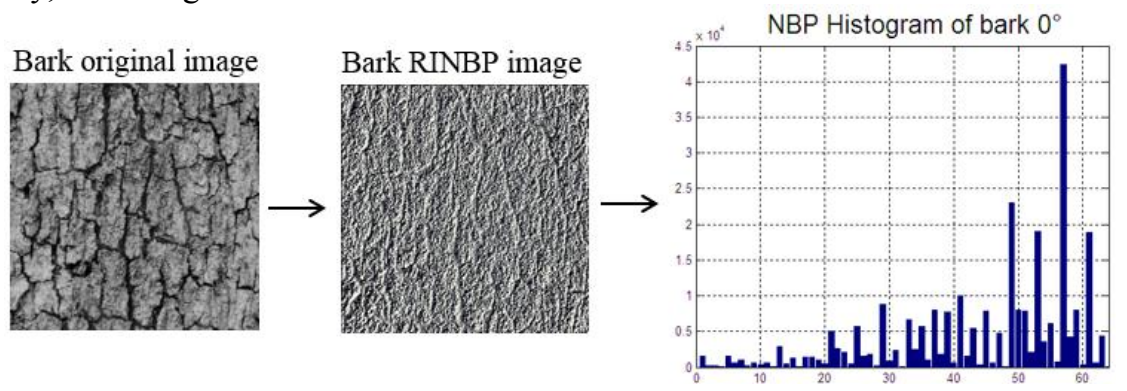

Fig. 2. Extraction of the NBP histogram.

Fig. 2. Illustrates the extraction of the NBP histogram. We can notice from the Fig. 2 that a small rotation in the input image would cause a change in the output NBP code. Thus, if the patterns are extracted from the input image and this image is rotated by an angel $\theta$, the extracted patterns will be different because the extraction starts always from the same point. That is the weakness of the classical LBP operator. In this work, we proposed a new system robust against the rotation problem.

\section{Proposed system}

In this section, we will explain the architecture of the proposed system. This system is robust against rotation and solve the rotation invariant problem. Some applications example will be illustrated and one illustration, which summarizes the proposed system, will be given.

The idea of the proposed system is to construct a model histogram of each texture. After that, compare the histogram of the researched texture with all model histograms to classify the texture. In order to solve the rotation problem, which is a fundamental problem on image processing and pattern recognition, we proposed to create a model histogram from each texture. First, two NBP histogram are extracted from two textured images, which have the same texture but different orientation. After that, a threshold histogram (Hist $t_{\text {th }}$ ) is calculated based on the two NBP histograms. The threshold histogram contains the minimum and the maximum value of each bin of the two histograms. Indeed, the Hist $t_{\text {th }}$ is the union of the two NBP histograms. This process is illustrated in Fig. 3. 


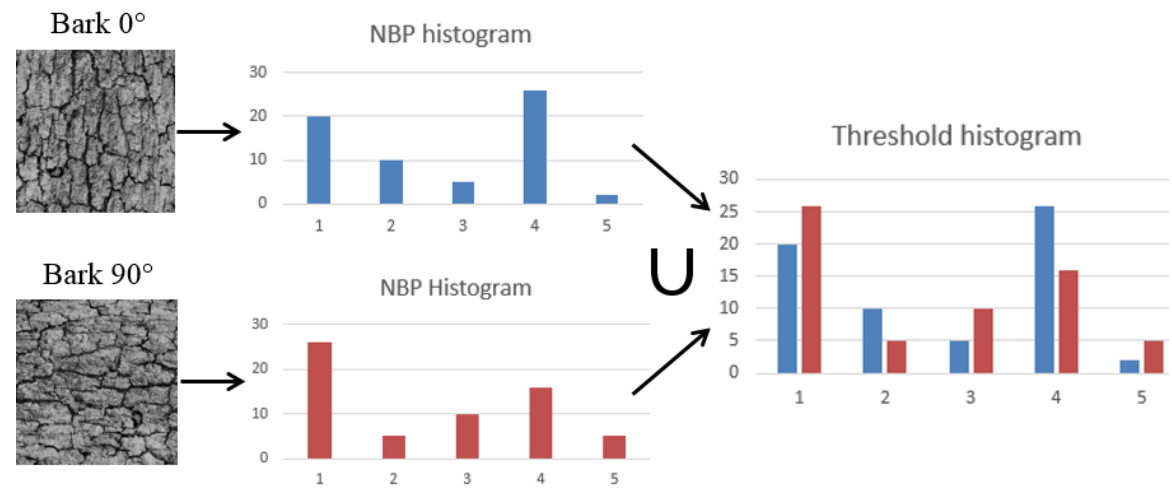

Fig. 3. Construction of the model histogram.

Fig. 3. Illustrates the construction of the model histogram of the Bark texture. The threshold histogram of the Bark texture is constructed using the angles 0 and 90 . Each bin of the threshold histogram, called model histogram, is an interval between the minimum and the maximum of the two NBP histogram of the two references images. Thus, a double threshold histogram is obtained and considered as a model of the texture.

In order to classify one query texture, a similarity distance is calculated between the NBP histogram of the query texture and the model histogram. The similarity vector is calculated following the formula 1 .

$$
\forall i \in[0,255]_{v} v(i)=\left\{\begin{array}{c}
1 \text { if hist }_{q}(i) \in \text { hist }_{\text {th }}(i) \\
0 \text { otherwise }
\end{array}\right.
$$

Where his $_{\mathrm{tq}}$ is the NBP histogram of the researched texture. Hist ${ }_{\mathrm{th}}$ is the model histogram. Finally, a binary vector $v$ is extracted where 1 means that the bin belongs to the interval of the model and 0 otherwise. The extraction of the similarity vector $v$ is illustrated in Fig. 4. 


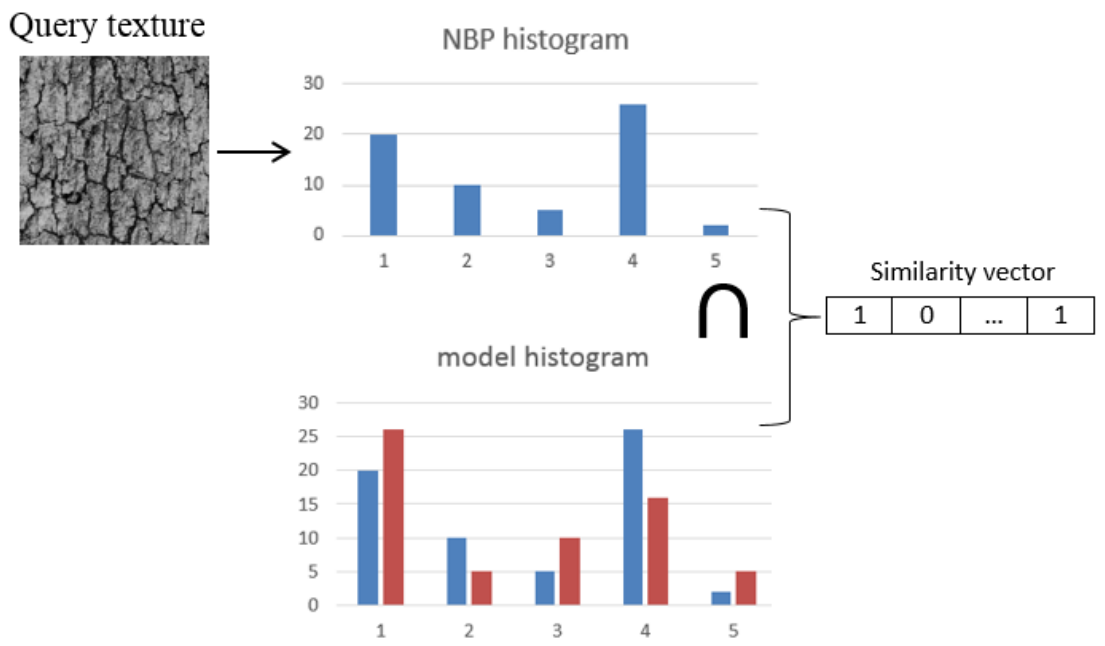

Fig. 4. Extraction of the similarity vector.

Figure 4 illustrates the extraction of the similarity vector $v$. First, the NBP histogram is extracted from the query texture. After that, the intersection between the NBP histogram and the model histogram is encoded by 1 , if the bin is in the double threshold, and 0 otherwise. The number of occurrence of the value 1 represents the similarity measure.

\section{Experimental results}

In this section, the evaluation of the proposed system will be illustrated. In order to evaluate the performances of our proposed system, we used textured images from Brodatz album database [13]. Brodatz album is a famous benchmark for textured images. In the experimentation, we used twelve textured images (bark, brick, bubbles, grass, leather, pigskin, raffia, sand, straw, water, weave and wood) illustrated in Fig. 5 . 

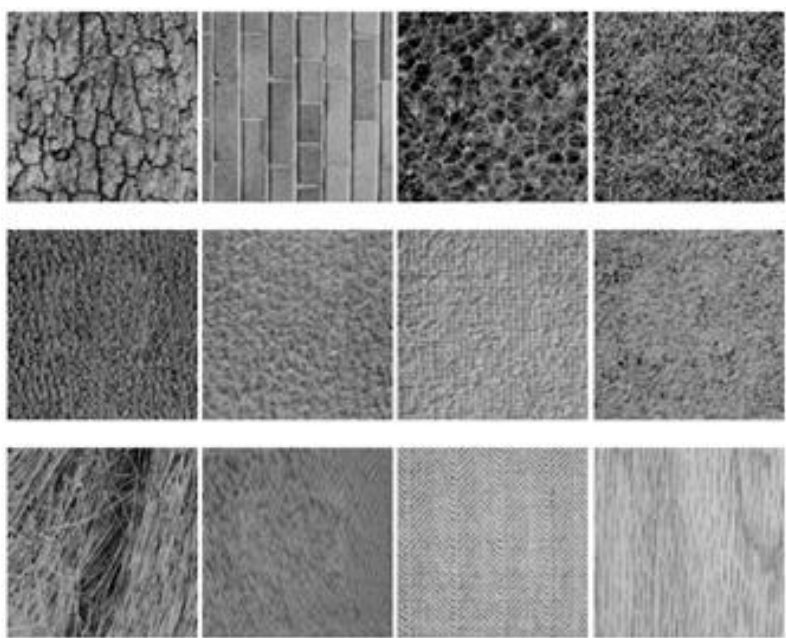

Fig. 5. Textured images from Brodatz album.

Each image is digitized at seven different rotation angles: 0, 30, 60, 90, 120, 150, and 200 degree. The size of the images are $512 \times 512$ pixels with 256 gray levels as illustrated in Fig. 6, which contains a total of 84 images (12 different images with their 7 rotations).
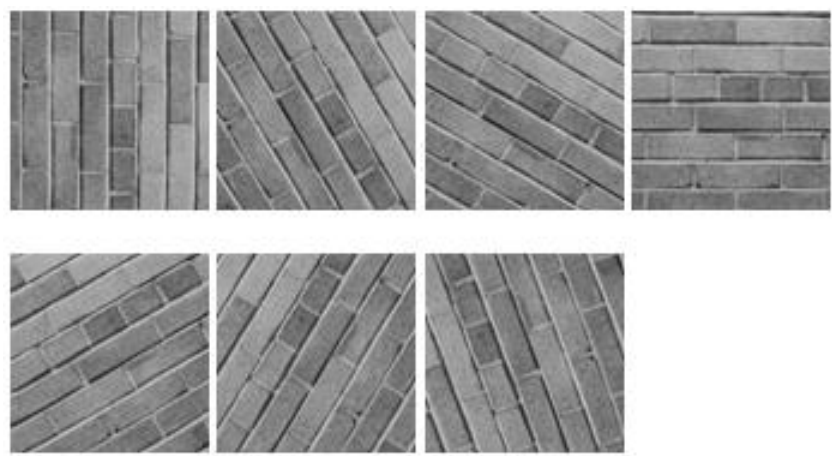

Fig. 6. Brick texture on seven orientation.

In order to construct the model database of the system, model histograms are extracted from each texture. Thus, we obtain twelve model histograms. A classification process is applied to classify the query textures. First, the NBP histogram of the query texture is extracted. After that, the similarity measure is calculated between the NBP histogram of the researched texture and all model histograms of the system. Finally, the query texture is classified according to the most similar model.

In the evaluation part, all textured images of the database are considered as query texture. So, 84 test images (twenty images and its seven different orientation). 
In order to compare the proposed system and the traditional system; the recognition averages of each texture are compared. The traditional texture classification system consider one textured image of each texture as reference. After that, the NBP histogram of the query texture is compared with all NBP histograms of the reference images and classified according to the most similar texture. The average recognition rate of each texture of the database (texture1 to texture 12) using the two recognition systems are illustrated in Table 1.

Table 1. Recognition rate of each texture.

\begin{tabular}{ccc}
\hline System & Traditional & Proposed \\
\hline Bark & 100,00 & 100,00 \\
Brick & 85,71 & 81,23 \\
Bubbles & 100,00 & 95,91 \\
Grass & 71,42 & 100,00 \\
Leather & 55,10 & 85,71 \\
Pigskin & 73,46 & 100,00 \\
Raffia & 51,02 & 100,00 \\
Sand & 100,00 & 100,00 \\
Straw & 42,85 & 95,26 \\
Water & 46,93 & 100,00 \\
Weave & 100,00 & 100,00 \\
Wood & 46,93 & 100,00 \\
Recognition Rate & $\mathbf{6 7 , 1 9}$ & $\mathbf{8 9 , 0 9}$ \\
\hline
\end{tabular}

Table 1 illustrates the average of the recognition rate of each texture. We can notice that few textures are better recognized using the traditional method. However, most of all textures are well recognized using the proposed system and better classified. The traditional system consider only one orientation as reference. In fact, the weaknesses of the traditional system (rotation) are improved in our method with the model histogram (Double threshold histogram). This allows us to analyze the image with different orientations. This represents the strength of our method. The obtained comparison results are also illustrated in Fig. 7. 


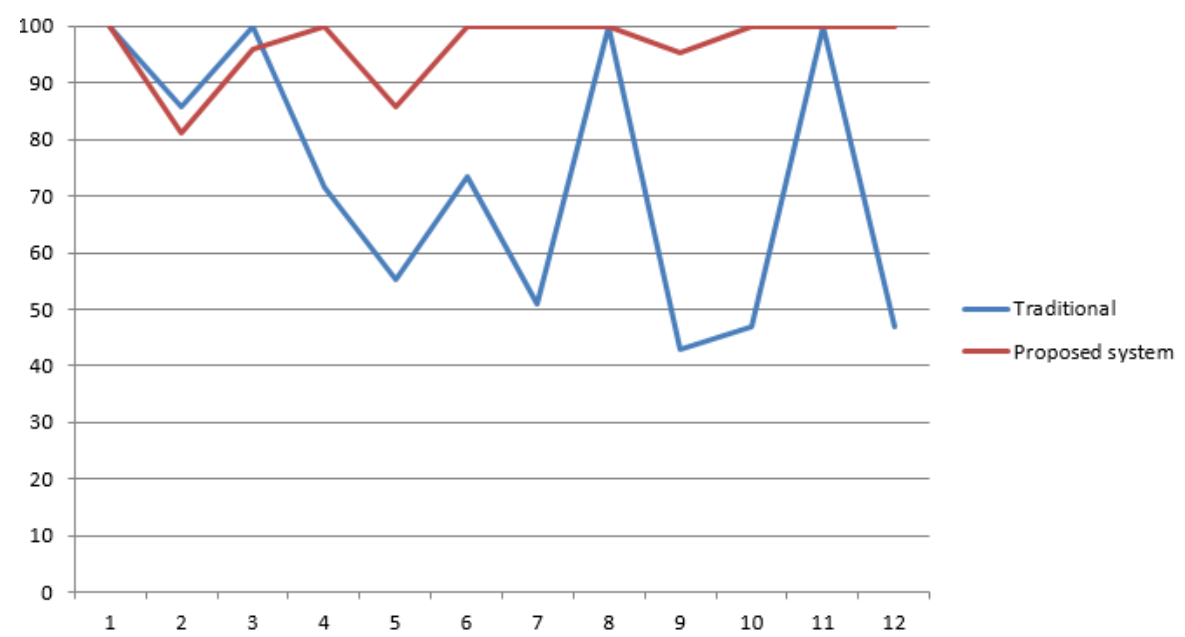

Fig. 7. Recognition rate using the traditional and proposed system.

We can notice in the Fig. 7 that the traditional system (blue histogram) gives a lower performance compared to the proposed method (red histogram). Thus, we can see that the results given by our method are better than the classical method.

From the experiments and based on these obtained results, the global rate of the classical method is $67,19 \%$ and the proposed method is $89,09 \%$. We can draw a conclusion that the proposed method, which is based on double threshold model histogram, is robust against rotation. Thus, the proposed system extract a robust model from the texture.

\section{Conclusion}

In this paper, we proposed a new rotation invariant system using the NBP method to extract models that describe the texture. First, the NBP method is applied on two textured images, which are in different orientation. After that, a double threshold histogram is calculated based on the two NBP histograms extracted from the two images and considered as model of the input texture. The model histogram is the union of the two NBP histograms. Finally, this process is applied on all textures of the database to extract models from each texture.

A similarity measure is calculated between the query texture and the model histograms. This measure is the intersection between the NBP histogram of the query texture and all model histograms. Each bin of the query texture histogram is encoded by 1 if its value is between the double threshold model histogram and 0 otherwise. Finally, a binary vector is extracted, which is the similarity vector. The query texture is classified according to the most similar vector. The obtained results show the efficiency of the proposed method compared to the traditional system. The robustness against rotation of the model histogram and the applied feature extraction method are the advantages of the proposed system. 
In future works, we will combine other approaches to get more information from the texture like multi resolution methods. We will also improve the classification and the similarity measure to improve the recognition rate. We will also study the behavior and the robustness of our approach applied on real textured images.

\section{References}

1. Richards, W and A Polit, "Texture matching", Kybernatic, 16, pp. 155 - 162, 1974.

2. Y. Baohua, H. Yuan, and C. Jiuliang, Combining Local Binary Pattern and Local Phase Quantization for Face Recognition, Biometrics and Security Technologies (ISBAST), pp. 51-53, March 2012

3. A.K. Jain, A. Ross, S. Prabhakar, "Fingerprint matching using minutiae and texture features", International Conference on Image Processing, vol. 3, pp. 282285, 2001

4. Hamouchene, I.; Aouat S. and Lacheheb, H., " Texture Segmentation and Matching Using LBP Operator and GLCM Matrix", Intelligent Systems for Science and Information, Springer International Publishing, vo. 542, pp. 389-407, 2014.

5. Harlick, R., "Statistical and structural approaches to texture", Proc. Of IEEE, vol. 67, no. 5, pp. 786-804, May 1979.

6. Hamouchene, I.; Aouat, S.; "A New Texture Analysis Approach for Iris Recognition", AASRI Conference on Circuit and Signal Processing (CSP 2014), Volume 9, Pages 2-7, 2014.

7. Hamouchene, Izem; Aouat, Saliha, "A cognitive approach for texture analysis using neighbors-based binary patterns," Cognitive Informatics \& Cognitive Computing (ICCI*CC), IEEE 13th International Conference on , pp.94,99, 18-20 Aug. 2014.

8. T. Ojala, M. Pietikäinen, and D. Harwood, "A Comparative Study of Texture Measures with Classification Based on Feature Distributions", Pattern Recognition, vol. 29, pp. 5159, 1996.

9. T. Ojala and M. Pietikäinen, "Unsupervised Texture Segmentation Using Feature Distributions", Pattern Recognition, vol. 32, pp. 477-486, 1999.

10. Zhenhua Guo; Lei Zhang; Zhang, D.; , "A Completed Modeling of Local Binary Pattern Operator for Texture Classification," Image Processing, IEEE Transactions on , vol.19, no.6, pp.1657-1663, June 2010.

11. Xueming, Q., Xian-Sheng, H. and Ping C., Liangjun, K. "An effective local binary patterns texture descriptor with pyramid representation",Pattern Recognition, Vol. 44, Issues 10-11, pp. 2502-2515, November 2011.

12. Baohua, Y., Yuan, H. and Jiuliang, C. "Combining Local Binary Pattern and Local Phase Quantization for Face Recognition “, Biometrics and Security Technologies (ISBAST), pp. 51-53, March 2012.

13. P. Brodatz, "Textures: A Photographic Album for Artists and Designers", Dover Publications, New York, 1966. 